\title{
Distribution of supercritical nucleation centers during the crystallization process in the presence of a flow as illustrated by the example of boron carbide-reinforced aluminum
}

\author{
M. A. Durnev ${ }^{1}$, E. D. Eidelman ${ }^{1,2}$ \\ ${ }^{1}$ St. Petersburg Chemical-Pharmaceutical Academy, \\ Professora Popova, 14, St. Petersburg, 197022, Russia \\ ${ }^{2}$ Ioffe Physico-Technical Institute, \\ Politechnicheskaya, 26, St. Petersburg, 194021, Russia \\ mad5245mail@gmail.com
}

PACS 05.20.Dd, 64.60.Qb, 64.70.kd

DOI 10.17586/2220-8054-2017-8-3-360-364

\begin{abstract}
The distribution of heavily post-critical nucleation centers in the nucleation kinetics subject to the macroscopic flow effect has been successfully obtained. Examples of rotary and convective motions during the centrifugal casting are considered. The obtained distribution has been confirmed by an experimental investigation of the composite material AK12 (boron carbide $\left(\mathrm{B}_{4} \mathrm{C}\right)$ ) crystallization.
\end{abstract}

Keywords: First-order phase transition, Distribution of supercritical nucleation centers, Crystallization in the presence of flow effect.

Received: 12 January 2017

Revised: 8 March 2017

\section{Introduction}

In the first-order phase transition, e.g., crystallization, two steps are identified. The first one [1] is the formation of critically sized nucleation centers in the metastable system, i.e. the size of nucleation centers is such that the system does not return to the initial one-phase state. The second step [2] is the evolution of post-critical big nucleation centers of a new phase. It is such quickly growing nucleation centers that accumulate, integrate, and absorb the metastable phase with small-sized nucleation centers.

This paper focuses on the second step, using the example of crystallization. The theory of this step of the nucleation process without the macroscopic flow effect in the metastable material is well known, but this theory is constructed using the example of vapor condensation (see, for example, review [3]). In recent years, the size distribution of supercritical nucleation centers and its time evolution were obtained within the framework of this theory $[4,5]$.

In connection with the use of centrifugal casting technologies with a high rotary speed of lingots, a problem arose when predicting the influence of the flow, first of all, rotation, will have on crystallization and, in a broader sense, on first order phase transitions. Certainly, flow effects of different kinds occur during the material treatment. For example, during extra-high-power energy flux application, different mechanisms of convection excitation are revealed [6]. However, it is the influence of rotation that seems to be of special interest.

The investigation of rotations influence is important for developing technologies for gradient spatiallyinhomogeneous composite material production. Due to spatial inhomogeneity, for example, surface hardening, such materials could potentially be used for manufacturing structures operating under extremely difficult conditions.

Centrifugal casting is a well-known practice of producing composite materials, in particular, aluminum-based ones [7]. Alternatively, additional reinforcing materials allow one to perform microscopic investigation of sections of solid castings, which indicate the state of melt precisely on the second step of nucleation.

In this work, the distribution of supercritical nucleation centers in the presence of flow effect, which was obtained theoretically, will be confirmed experimentally using the example of a composite produced by centrifugal casting from the aluminum alloy AK12 and boron carbide particles.

\section{Distribution of supercritical nucleation centers in the presence of flow effect}

Let us consider the mixture of the main material (in this experiment, it will be aluminum alloy AK12) and additional material (in this experiment, it will consist of boron carbide particles). Nucleation [4] occurs mainly at 
condensation centers around the particles of the additional material. At the post-critical step, when no macroscopic flow effects exist, the function of nucleation centers distribution [5] may be written as a Gaussian distribution:

$$
G=\Theta(t) \frac{1}{\sqrt{D t}} \exp \left(-\frac{\left(d-d_{0}\right)^{2}}{4 D t}\right) .
$$

Here, $D$ is the coefficient of small nucleation centers diffusion, actually, the coefficient of molecular diffusion in liquid melt; $t$ is the time from the beginning of the process; $d-d_{0}$ is the degree of post criticality, which determines the deviation of the large nucleation center size $d$ from the critical nucleation center size $d_{0}$ under conditions displaying a high degree of post criticality, $d \gg d_{0}$. The value $\Theta-$ is the amplitude, which does not depend on the nucleation center size.

From the nucleation pattern, it is obvious that the probability of a small nucleation centers being absorbed by a big one is proportional to the coefficient $D$. On the other hand, from the dimension considerations, it follows that $D \sim v S / R \approx C_{1} v R$, where $S$ is the surface area and $R$ is the size of the big nucleation center. The order of magnitude of the constant $C_{1}$ is one. Most importantly, $D \sim v$ rms velocity of small nucleation centers.

In the presence of a flow effect in the liquid melt, it is the flow velocity that becomes the main part of relative motion velocity of nucleation centers.

In the case of convective motion excitation, the flow velocity is proportional to the square root of overcriticality, the amount by which the number determining convection exceeds the critical value at which convection is excited [6].

During rotation, a large nucleation center tends to proceed in straight-line motion, but the viscous friction force prevents it. It is easy to find out that in this case the velocity caused by rotation with the angular speed $\omega$ of small nucleation centers located at the distance $r$ from the rotation axis will be:

$$
v=\omega r \exp \left(-\frac{9 \eta t}{2 \rho R^{2}}\right)
$$

where $\eta$ and $\rho$ are viscosity and liquid density correspondingly. This directly follows from the law of motion of a particle in a rotating melt by the action of Stokes force $6 \pi \eta v R$. Certainly, it is proposed here that a small nucleation center is originally a sphere. Another form of nucleation center may correspond to a somewhat different numerical coefficient in exponent, but qualitatively, the result will not change.

Thus, in the function of large nucleation centers size distribution (spectrum) in the presence of rotation the diffusion coefficient should be replaced with the following value:

$$
D=C_{2} \omega r \exp \left(-\frac{9 \eta t}{2 \rho R^{2}}\right)
$$

Here, $C_{2}$ is another constant, but it is as well as $C_{1}$, has the same order of magnitude.

These results will be experimentally confirmed in the next section.

It may be considered that it is the boron carbide particles that are the nucleation centers and their distribution on the material sample after crystallization (Fig. 1) reflects the distribution of supercritical nucleation centers before the phase transition. During the phase transition, the nucleation centers accrete and their growth is limited only by the size of melt. During rapid rotation, centrifugal forces support the growth mainly from the axis of rotation to the lingot. Hence, it follows that the size of the nucleation center $d$ turns into the distance between the nucleation center position and the external boundary of the melt.

The distribution (1) is Green's function of the kinetic equation determining the growth of supercritical nucleation centers $[4,5]$. To obtain the distribution $D$ of boron carbide in the sample, it is necessary to put in it the time of phase transition $t_{f}$, which is much less than the typical fall time $2 \rho R^{2} / 9 \eta$, and put in (1) the value of $D$, which is determined by the flow in accordance with formula (3) for supercritical nucleation centers before the phase transition.

Let us note the supercritical nucleation centers distribution before the phase transition as a function of distance $x$ measured from the external wall of the sample or, which is the same, from the lingot. We have:

$$
P(x)=A \exp \left(-\frac{x^{2}}{4 \sigma^{2}}\right) .
$$

Here, $A$ is the amplitude, which has the form:

$$
A=\frac{B}{\sqrt{\omega r R t_{f}}} \exp \left(-\frac{9 \eta t_{f}}{8 \rho R^{2}}\right) .
$$

This value contains the parameter $B$ which depends upon the buoyancy forces. 


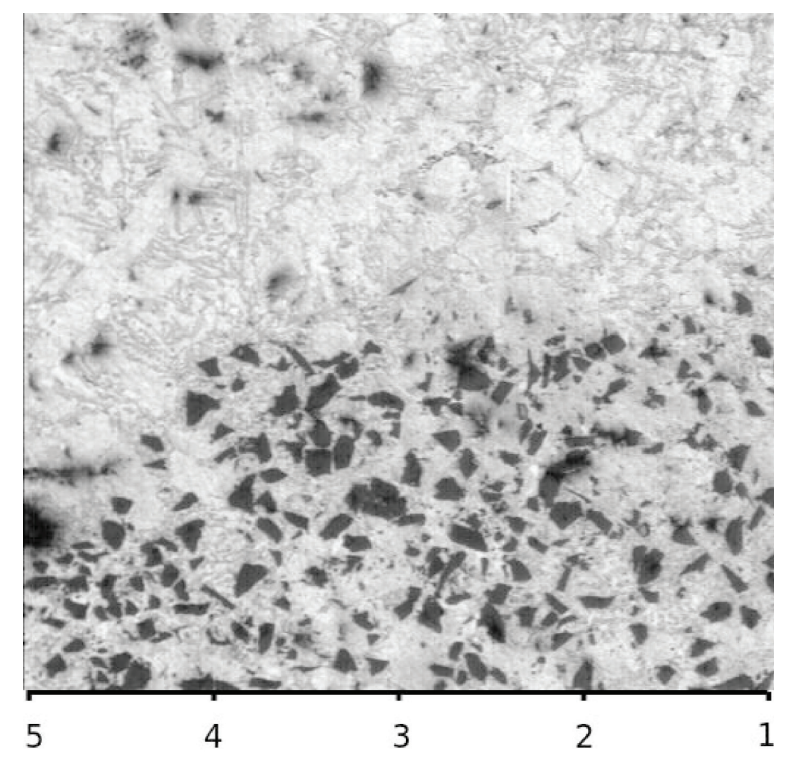

FIG. 1. Typical microstructure of a sample of composite of alloy AK12 with addition of $10 \%$ $\mathrm{B}_{4} \mathrm{C}$. Regions encircled with dark ovals are the reinforcing additional material. The distance from the external wall is indicated (in $\mathrm{mm}$ ) from right to left

The standard deviation in distribution

$$
\sigma=\sqrt{\omega r R t_{f}} \exp \left(-\frac{9 \eta t_{f}}{8 \rho R^{2}}\right)
$$

may be considered to be independent of the buoyancy force. The value $C_{2}$ from (3) is included in value $t_{f}$, defined the most roughly. The derived above relation $D \sim v R$ was used for obtaining of expressions (4) - (6).

\section{Design of the material produced by centrifugal casting method from AK12 and boron carbide particles}

As previously mentioned, aluminum alloy AK12 with density in the liquid state $\rho_{a} \approx 2.67 \mathrm{~g} / \mathrm{cm}^{3}$ was used as the primary material. Particles of boron carbide with the density of powder $\rho_{b} \approx 2.5 \mathrm{~g} / \mathrm{cm}^{3}$ were used as the additional material. These particles had the shape of a sphere with the diameter $\leq 60 \mu \mathrm{m}$ and accounted for $10 \%$ of the mixture weight.

Samples shaped as bushings with $r_{\min } \leq r \leq 93 \mathrm{~mm}=r_{\max }$ were produced using the centrifugal casting method with the angular speed $\omega=40 \pi \mathrm{rad} / \mathrm{s}$. The bushings were slit by element and were investigated by the Leica microscope. The results are presented in Fig. 1.

Recalculation in the distribution of boron carbide particles in layers on three levels of casting height (60$90 \mathrm{~mm}$ ) is presented in Figs. 2-4.

The casting conditions were the same for all samples: the heating temperature was $210 \pm 10{ }^{\circ} \mathrm{C}$ for the lingot and $750 \pm 10^{\circ} \mathrm{C}$ for the melt.

\section{Discussion}

Experimental data were processed according to the ideas developed in Sect. 2. Continuous lines are drawn in Figs. 2-4 using formulae (4) in the least-squares technique. The curves have the following parameters: line in Fig. 2, corresponding to the top of the sample, has the amplitude $A=A_{1}=30 \%$ and the standard deviation $\sigma=\sigma_{1}=0.88 \mathrm{~mm}$; line in Fig. 3, corresponding to the centre (middle) of the sample, has the amplitude $A=19.6 \%$ and $4 \sigma^{2}=9.6 \mathrm{~mm}^{2}$; line in Fig. 4, corresponding to the bottom of the sample, has the amplitude $A=20.6 \%$ and $4 \sigma^{2}=7.4 \mathrm{~mm}^{2}$. As can be seen from Fig. 1, the reinforcing particles are concentrated in the middle and bottom of the sample, therefore lines on Figs. 3, 4, corresponding to the middle and the bottom, sharply differ from the line for the top part (Fig. 2) and slightly differ from each other. For simplicity, these two lines will be considered as one with the parameters $A=A_{2}=20 \%$ and $\sigma=\sigma_{2}=1.46 \mathrm{~mm}$.

It is important that the phase transition time for supercritical nucleation centers calculated by formula (6) is much less than the characteristic fall time at $\eta \approx 0.0032 \mathrm{~Pa} \cdot \mathrm{s}$ and $R \gg 60 \mu \mathrm{m}$,

$$
8 \rho_{a} R^{2} / 9 \eta \geq 1 \mathrm{~s}
$$




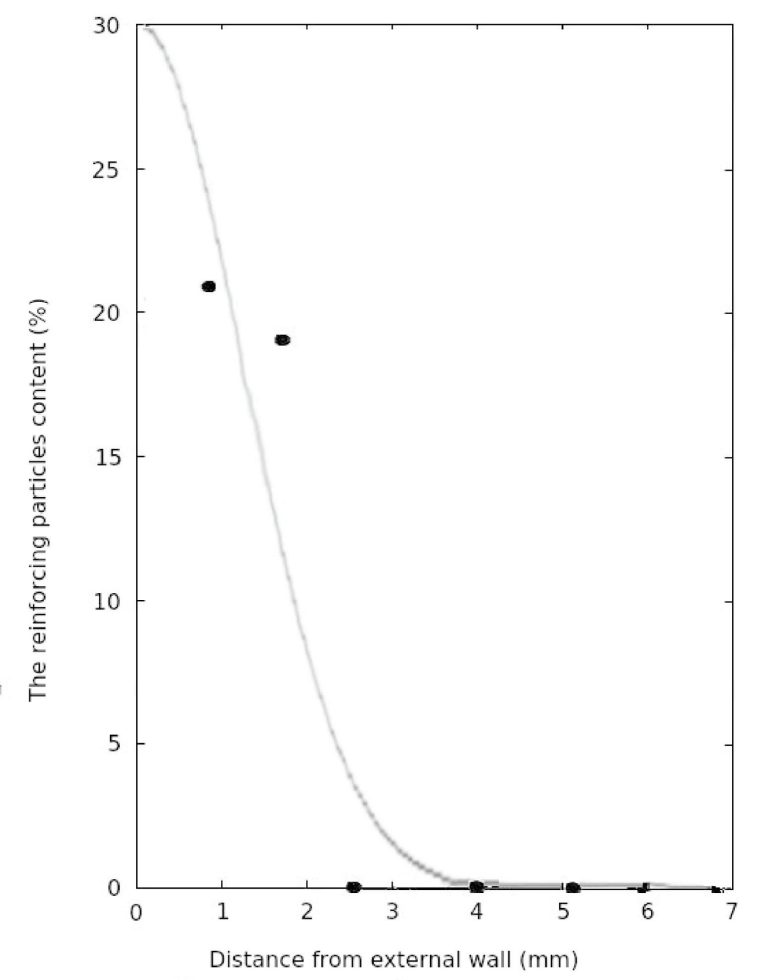

FIG. 2. Typical distribution of boron carbide particles in samples of the same material as in Fig. 1 in the top part of the bushing. The continuous line is the distribution function

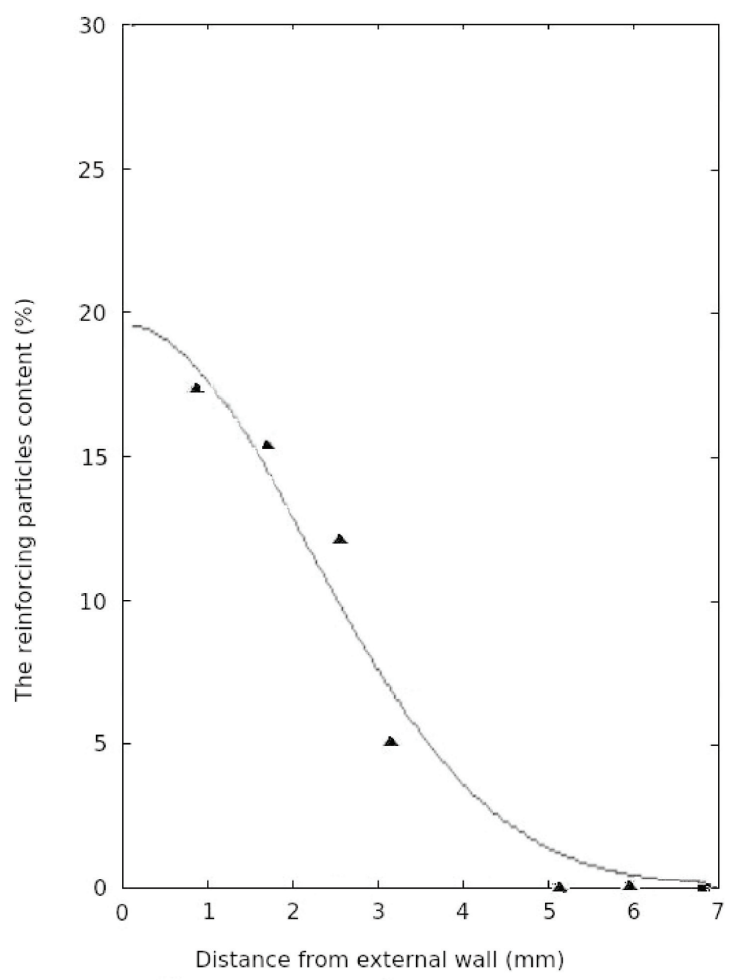

FIG. 3. Typical distribution of boron carbide particles in samples of the same material as in Fig. 1 in the middle part of the bushing. The continuous line is the distribution function

The distributions presented in Figs. 2-4 are in good agreement with the experimental results obtained by processing first-hand observation data in Fig. 1, if the nucleation center size achieves the crystallization region size, which is approximately equal to the sample thickness $R \approx 10 \mathrm{~mm}$, in a phase transition time, which is approximately several milliseconds. More precisely: in the top of the bushing, where there is less reinforcing material, this time is $t_{f} \approx 3 \mathrm{~ms}$, but in the middle and the bottom parts, where there is more reinforcing material, it is $t_{f} \approx 2 \mathrm{~ms}$.

Now, it is easy to determine from (5) the parameter $B$ depending on buoyancy forces. We find: $B_{1}=4.2 \mathrm{~mm}$; $B_{2}=3.4 \mathrm{~mm}$. Certainly, all the determined values are no more than qualitative (order-of-magnitude) estimates which may depend on many neglected factors, for example, viscosity increases abruptly at the time of phase transition [8]. Tribological properties also depend upon the contact surface properties of the structures which correspond to different nucleation centers [9]. However, these estimates will certainly help choose the quantity and quality of the reinforcing material necessary for producing the material with tailored properties.

\section{Conclusion}

It was proved that the distribution (spectrum) of large supercritical nucleation centers during nucleation, at least, during crystallization, with the flow effect may be obtained from the distribution determined as a result of solution of the kinetic equation [5] without macroscopic flow effects. For this purpose, the value replacing the diffusion coefficient and determined by the macroscopic flow velocity should be included in the distribution of supercritical nucleation centers. The phase transition time was estimated here at about several milliseconds. As expected, the presence of reinforcing particles, which are the primary critical nucleation centers, accelerates the phase transition.

It has been demonstrated that additional material $\mathrm{B}_{4} \mathrm{C}$ can increase the hardness of near-surface layers using the centrifugal casting method and, consequently, this method can be used for designing materials with tailored structural properties. 


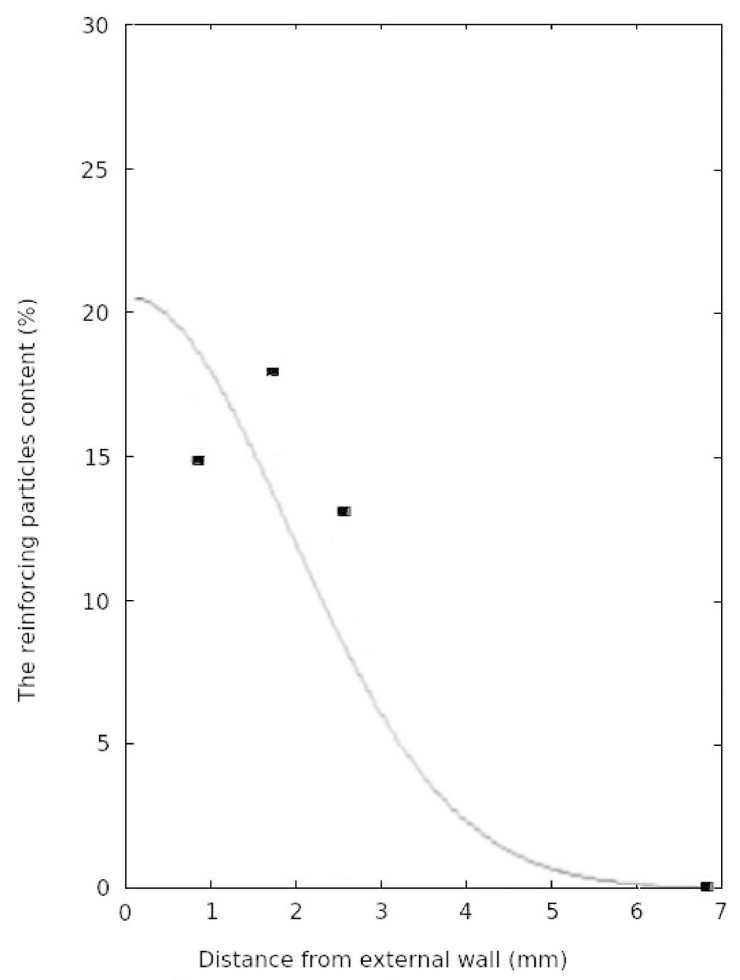

FIG. 4. Typical distribution of boron carbide particles in samples of the same material as in Fig. 1 in the bottom part of the bushing. The continuous line is the distribution function

As it was postulated, success was achieved in creating surface layers with a higher concentration of the reinforcing (additional) phase. All this suggests that a gradient composite material can be created (designed) using centrifugal casting methods with a high rotation velocity.

\section{Acknowledgements}

The authors are much obliged to M.L. Heifgetz for drawing our attention to the investigation of centrifugal casting. We also thank Yu. S. Alekseeva for her kind assistance. E. D. Eidelman is grateful to the RSF (Russian Science Foundation) for support (Grant 16-19-00075).

\section{References}

[1] Landau L.D., Lifshitz E.M. Statistical Physics, Third ed., Part 1. Course of Theoretical Physics, 5, §162, Butterworth-Heinemann, 1980.

[2] Lifshitz E.M., Pitaevskii L.P. Physical Kinetics. Course of Theoretical Physics, 10, §§99-102, Pergamon Press, Oxford, 1981.

[3] Kuni F.M., Shchekin A.K., Grinin A.P. Theory of heterogeneous nucleation for vapor undergoing a gradual metastable state formation. Phys. Usp., 2001, 44, P. 331-370.

[4] Kurasov V.B. Advances in the first order phase transitions. II. VVM Publishing Ltd., St. Petersburg, 2013.

[5] Kurasov V.B. Distribution of supercritical nuclei in nucleation kinetics. Tech. Phys. Lett., 2015, 41, P. 348-351.

[6] Eidelman E.D. Excitation of an Electric Instability by Heating. Physics Uspechi, 1995, 38 (11), P. 1068-1083.

[7] Kevorkijan V. Functionally graded aluminum-matrix composites. American Ceramic Society Bulletin, 2003, 82 (2), P. 60-64.

[8] Vityaz P.,A., Senyut V.T., et al. Technological fundamentals of nano-structured composite synthesisased on aluminum alloys. Science intensive technologies in mechanical engineering, 2016, (8), P. 3-12.

[9] Eidelman E.D. Estimation of the contact area of solids by electrothermal analogy. Nanosystems: physics, chemistry, mathematics, 2015, 6 (4), P. $547-550$. 